\title{
Imaging for biopsies
}

\section{Musaad AlBalood*, Mohammed Almeshal}

\author{
Imam Muhammed Ibn Saud Islamic University, Riyadh, KSA
}

Received: 01 October 2018

Accepted: 18 October 2018

\section{*Correspondence:}

Dr. Musaad AlBalood,

E-mail: msb_1414@hotmail.com

Copyright: (c) the author(s), publisher and licensee Medip Academy. This is an open-access article distributed under the terms of the Creative Commons Attribution Non-Commercial License, which permits unrestricted non-commercial use, distribution, and reproduction in any medium, provided the original work is properly cited.

\begin{abstract}
Positron emission tomography/computed tomography (PET/CT) imaging technique is considered to be an advanced technique that emerged recently and has been successfully used to guide several procedures like obtaining biopsies and ablating tumors. In this review, we will thoroughly discuss the indications, uses, and advantages of guiding biopsies with PET/CT techniques, with main focus on the field of oncology. PET/CT techniques are considered to be emerging imaging modalities that have significantly improved diagnostic accuracy of biopsies. The most important advantage of using PET/CT imaging to guide biopsies is its ability to provide accurate visualization of the lesion, with immediate assessment of the procedure following its end. On the other hand, this technique still has some limitations. Most importantly, the significant exposure to radiation to both the patient and the physician.
\end{abstract}

Keywords: Biopsy, PET/CT, Imagings techniques

\section{INTRODUCTION}

Positron emission tomography/computed tomography (PET/CT) imaging technique is considered to be an advanced technique that emerged recently and has been successfully used to guide several procedures like obtaining biopsies and ablating tumors. These procedures guided by PET/CT mainly depend on previously obtained PET/CT retrospective fusion into intra-procedural images. ${ }^{1}$

The limitation of this technique has been the imprecise registration of images, which is caused by the possible different positions in which an intra-procedural CT image could be obtained. The phase of the breathing cycle in which the image is obtained could also play a role in the accuracy of this images. ${ }^{1}$ On the other hand, recent advances in the PET/CT technologies have led to improved integration of interventional units of CT with the machines. Therefore, allowing the opening of routes to directly performing procedures with PET/CT guidance. ${ }^{2}$ This last technique is not as widely available as the previous ones, and it usually can only be done in specialized PET/CT interventional suites. ${ }^{3}$

In this review, we will thoroughly discuss the indications, uses, and advantages of guiding biopsies with PET/CT techniques, with main focus on the field of oncology.

\section{METHODS}

We did a systematic search for imaging and biopsies using PubMed search engine (http://www.ncbi.nlm.nih.gov/) and Google Scholar search engine (https://scholar.google.com). Our search also looked for indications and uses of PET/CT techniques in biopsies. All relevant studies were retrieved and discussed. We only included full articles.

The terms used our systematic search included: imaging modalities, radiology, imaging-guided biopsy, diagnostic biopsies, imaging-guided procedures, and PET/CT imaging techniques. 


\section{INDICATIONS OF PET/CT GUIDED PROCE- DURES}

PET/CT imaging is generally indicated to guide in biopsies of bony or visceral lesions, as these lesions will have intense activity metabolically, which can be easily visualized on PET/CT, but poorly on other modalities like ultrasound, MRI, and CT. On the other hand, ultrasound, MRI, CT, and similar imaging modalities are usually used in the guidance of superficial or percutaneous biopsies. ${ }^{2}$

PET/CT imaging modalities can also be used to guide biopsies of lesions that had been previously biopsied with other techniques (ultrasound or CT) and reached inconclusive results with high clinical suspicion. These lesions must, however, have high metabolic activity for this technique to work. ${ }^{2}$

\section{ADVANTAGES OF PET/CT GUIDED PROCE- DURES}

The most important advantage of using PET/CT imaging techniques when guiding biopsies is allowing for combination of both functional imaging and anatomical imaging to be able to precisely detect and identify lesions that were not detected or visualized using other imaging modalities. This is mainly achieved in PET/CT by administrating radiotracer agonists before initiating the procedure, leading to significantly increasing toe bioavailability of the radiotracer and boosting the visualization of the target. $^{2}$

Generally, radiotracers can have a long half-life that allows them to persist in the lesion for over few hours. In their study, Sainani concluded that the PET/CT imaging was associated with a $24.6 \%$ increase in the uptake of the radiotracer by the lesion during biopsies. ${ }^{4}$ This is considered an important advantage of using PET/CT imaging over other possible techniques like ultrasound and MRI, which do not have this advantage, and can only show temporary enhancement. This prolonged uptake of radiotracers will further lead to many subsequent advantages including. ${ }^{5}$

- The continuous visualization of the lesion throughout the procedure, which is also extremely important in interventional procedures (like ablation) and not only biopsies. $^{5}$

- The ability to visualize the lesions even if there are adverse conditions leading to obscuring the lesion. These include the presence of hemorrhages, the presence of atelectasis, and the displacement of the target. $^{6}$

- The precise targeting and biopsying big lesions. This can also be useful while performing imaging-guided ablation. $^{6}$

- The ability to assess the rate of success of the technique following the biopsy. For example, performing a PET scan on the biopsied tissue will be able to prove the fixation of the radiotracer in the specimen, leading to assertion of the success of the technique. 7 This success assessment can also be performed following PET/CT procedures, which, however, could me more challenging than assessing the success of biopsies. ${ }^{4}$

\section{DISADVANTAGES OF PET/CT GUIDED PROCE- DURES}

Despite all advantages that PET/CT imaging carries, it still has several limitations and concerns. The main concern is the radiation exposure that patients get when undergoing PET/CT guided biopsy, which could be considered significant and unnecessary. This would be of special importance in pregnant women, for example, who must not obtain a biopsy under the guidance of PET/CT. It is estimated that the PET/CT imaging required to guide a biopsy will put the patient under exposure of a radiation dose of $370 \mathrm{MBq}{ }^{7}$ Moreover, some other PET/CT guided procedures may carry a risk of higher exposures like performing multiple ablations simultaneously. ${ }^{8}$

Unfortunately, these high doses of radiation cannot be reduced. Therefore, other approaches should be made to decrease radiation-associated harm. These approaches include sufficient hydration and complete voiding before the procedure, which will help excrete the radiotracer materials more easily and, thus, decrease the dose and its associated harm.

Some have attempted the use of lead aprons to decrease the risk of radiation. These aprons are generally used by radiologists and are effective in decreasing the radiation exposure. However, when it comes to PET/CT guided procedures, aprons have not been found effective in opposing the damaging effects of photons that are produced from PET/CT. Therefore, they have not been recommended for use during PET/CT guided biopsies. However, when the co-use of CT-fluoroscopy is applied, the use of aprons becomes beneficial. ${ }^{8}$

Apart from aprons, some other techniques have been successfully used for the protection against radiation exposure during a PET/CT guided biopsy. Ryan and colleagues performed a study to assess the exact amount of radiation exposure that is associated with both biopsies and ablation procedures guided with PET/CT along with factors associated with this exposure. They concluded that both the patient and the medical staff get significant amount of radiation exposure during these procedures. This exposure was higher during long procedures. They recommended that the medical staff should decrease their contact with the patient to decrease their radiation exposure as possible. ${ }^{8}$ Others recommend the use of navigational systems to decrease the radiation exposure. ${ }^{9}$

Generally, we can determine the radiotracer dose that should be administrated based on two measurements: the first portion which will need to target the lesion, and the 
second portion which needs to stay efficient throughout the procedure. ${ }^{2}$

\section{CURRENT APPLICATIONS AND EVIDENCE FOR USING PET/CT GUIDED BIOPSIES}

The use of PET/CT imaging techniques to guide biopsies have been increasing especially during histopathological sampling of bony and visceral lesions. ${ }^{10}$ Cornelis et al, performed a study on 106 patients who underwent PET/CT guided biopsies that were obtained from bones, liver, lungs, abdomen, and soft tissues. They found that PET/CT guided biopsies were associated with a $100 \%$ overall success rate in diagnosing the lesions. The sensitivity of PET/CT guided biopsying reached 100\% with no false negative results. The accuracy for detecting malignancy was similar for different locations, with significant difference between organs. The size of the lesion was also not associated with diagnostic accuracy, with smaller lesions and larger lesions being detected and diagnosed in the same accuracy. The overall complications and adverse events rate following a PET/CT guided biopsy was less than $4 \%$. These adverse events included pneumothorax, which occurred in three of the included patients, and hematoma of the liver, which occurred in only one patient and was selflimiting. ${ }^{10}$

Another study was conducted by Guo and their team where they assessed the efficacy and safety of bone biopsies guided by PET/CT in 51 patients with lung cancer metastatic to the lung. They also founded the sensitivity of PET/CT guided biopsies was $100 \%$, with an accuracy that reached $96 \%$. $^{3}$

Cerci et al also conducted a prospective study where they evaluated 181 patients who will undergo biopsies guided by PET/CT and compared them with other 142 patients who will undergo biopsies guided by standard CT techniques. They found no significant differences between the two groups regarding safety and complications rates. ${ }^{11}$

\section{CONCLUSION}

$\mathrm{PET} / \mathrm{CT}$ techniques are considered to be emerging imaging modalities that have significantly improved diagnostic accuracy of biopsies. Since their introduction, they have led to significant advances in the field of interventional oncology along with other fields. This importance come from their ability to target lesions that have low conspicuity or absent conspicuity when they are visualized using other modalities, like ultrasound, MRI, and CT. The most important advantage of using PET/CT imaging to guide biopsies is its ability to provide accurate visualization of the lesion, with immediate assessment of the procedure following its end. On the other hand, this technique still has some limitations. Most importantly, the significant exposure to radiation to both the patient and the physician. Further studies should be evaluated to assess the cost-effectiveness of using such techniques for guiding biopsies.

Funding: No funding sources

Conflict of interest: None declared

Ethical approval: Not required

\section{REFERENCES}

1. Lei P, Dandekar O, Widlus D, Shekhar R. Incorporation of preprocedural PET into CT-guided radiofrequency ablation of hepatic metastases: a nonrigid image registration validation study. J Digit Imaging. 2010;23:780-92.

2. Cazzato RL, Garnon J, Ramamurthy N, Tsoumakidou G, Imperiale A, Namer IJ, et al. 18FFDOPA PET/CT-guided radiofrequency ablation of liver metastases from neuroendocrine tumours: technical note on a preliminary experience. Cardiovasc Intervent Radiol. 2016;39:1315-21.

3. Guo W, Hao B, Chen H, Zhao L, Luo Z, Wu H, et al. PET/CT guided percutaneous biopsy of FDGavid metastatic bone lesions in patients with advanced lung cancer: a safe and effective technique. Eur $\mathrm{J}$ Nucl Med Mol Imaging. 2017;44:25-32.

4. Sainani NI, Shyn PB, Tatli S, Morrison PR, Tuncali K, Silverman SG. PET/CT-guided radiofrequency and cryoablation: is tumor fluorine- 18 fluorodeoxyglucose activity dissipated by thermal ablation? J Vasc Interv Radiol. 2011;22:354-60.

5. Ryan ER, Sofocleous CT, Schoder H, Carrasquillo JA, Nehmeh S, Larson SM, et al. Split-dose technique for FDG PET/CT-guided percutaneous ablation: a method to facilitate lesion targeting and to provide immediate assessment of treatment effectiveness. Radiology. 2013;268:288-95.

6. Shyn PB. Interventional positron emission tomography/ computed tomography: State-of-theart. Tech Vasc Interv Radiol. 2013;16:182-90.

7. Maybody M, Grewal RK, Healey JH, Antonescu CR, Fanchon L, Hwang S, et al. Ga-68 DOTATOC PET/CT Guided Biopsy and Cryoablation with Autoradiography of Biopsy Specimen for Treatment of Tumor-Induced Osteomalacia. Cardiovasc Intervent Radiol. 2016;39(9):1352-7.

8. Ryan ER, Thornton R, Sofocleous CT, Erinjeri JP, Hsu M, Quinn B, et al. PET/CT guided interventions: Personnel radiation dose. Cardiovasc Intervent Radiol. 2013;36:1063-7.

9. Grasso RF, Faiella E, Luppi G, Schena E, Giurazza F, Del Vescovo R, et al. Percutaneous lung biopsy: comparison between an augmented reality CT navigation system and standard CT-guided technique. Int J Cars. 2013;8:837-48.

10. Cornelis F, Silk M, Schoder H, Takaki H, Durack JC, Erinjeri JP, et al. Performance of intraprocedural 18-fluorodeoxyglucose PET/CTguided 
biopsies for lesions suspected of malignancy but poorly visualized with other modalities. Eur J Nucl Med Mol Imaging. 2014;41:2265-72.

11. Cerci JJ, Tabacchi E, Bogoni M, Delbeke D, Pereira $\mathrm{CC}$, Cerci RJ, et al. Comparison of CT and PET/CT for biopsy guidance in oncological patients. Eur J Nucl Med Mol Imaging. 2017;44:1269-74.

Cite this article as: AlBalood M, Almeshal M. Imaging for biopsies. Int J Community Med Public Health 2018;5:4988-91. 